\title{
A Case of Childhood Vitrectomy Performed for Dense Vitreous Hemorrhage Secondary to Leukemia Therapy and Tumor Lysis Syndrome
}

\author{
Takashi Kudo Yukihiko Suzuki Tomomi Metoki Mitsuru Nakazawa \\ Department of Ophthalmology, Hirosaki University Graduate School of Medicine, \\ Hirosaki, Japan
}

\section{Key Words}

Leukemia · Pediatric surgery · Tumor lysis syndrome · Vitrectomy · Vitreous hemorrhage

\begin{abstract}
Purpose: To report a case of vitrectomy performed in a child with dense massive vitreous hemorrhage due to secondary acute myelogenous leukemia (AML) and tumor lysis syndrome. Case: A 4-year-old boy with clear-cell renal cell carcinoma was successfully treated with chemotherapy in 2011. However, in May 2012, he developed secondary AML. Although he was treated with combined chemotherapy and radiation, tumor lysis syndrome occurred with renal and heart failure complications. After an ultrasound examination by pediatricians found bilateral subretinal protrusions, he was referred to our clinic. Fundus examinations confirmed that the protrusions were bilateral subretinal or choroidal hemorrhages. A few weeks later, dense vitreous hemorrhages occurred bilaterally, and he completely lost vision in both eyes. Electroretinograms were extinguished in both eyes. After improvement of his general condition, we performed a 25-gauge vitrectomy combined with lens extraction in his left eye in December 2012. After removal of the vitreous hemorrhage, we found the subretinal hemorrhage had already been absorbed, leaving a mottled fundus color. However, the optic disc was not pale. Nine months after the surgery, his best-corrected visual acuity finally improved to 0.1. Conclusion: We successfully treated a case of severe vitreous hemorrhage secondary to leukemia therapy and tumor lysis syndrome using 25 -gauge vitrectomy. This procedure may be safe and effective to perform, even in children with complications.
\end{abstract}

(C) 2015 S. Karger AG, Basel

KARGER 125/s $\quad \begin{aligned} & \text { Mitsuru Nakazawa, MD, PhD } \\ & \text { Department of Ophthalmology } \\ & \text { Hirosaki University Graduate School of Medicine } \\ & \text { 5 Zaifu-cho, Hirosaki 036-8562 (Japan) } \\ & \text { E-Mail mitsuru@cc.hirosaki-u.ac.jp }\end{aligned}$


Kudo et al.: A Case of Childhood Vitrectomy Performed for Dense Vitreous Hemorrhage Secondary to Leukemia Therapy and Tumor Lysis Syndrome

\section{Introduction}

Massive vitreous hemorrhage is not infrequently encountered in clinical practice in patients who develop leukemia and are treated with chemotherapy. Vitrectomy is indicated for vitreous hemorrhage, provided the patient's general condition is good enough to undergo the procedure. However, to the best of our knowledge, there have been no cases reported in the literature regarding the use of vitrectomy in a child with massive vitreous hemorrhage due to leukemia or secondary to its treatment. We recently evaluated and performed vitrectomy in a child with severe massive vitreous hemorrhage due to secondary acute myelogenous leukemia (AML) associated with disseminated intravascular coagulation (DIC) and tumor lysis syndrome. Herein, we report on the vitrectomy and the outcome of the patient after the procedure.

\section{Case Report}

A tumorous mass at the right lateral side of the abdomen of a 4-year-old boy was detected in April 2011. He was diagnosed as having right kidney tumor and was subsequently hospitalized in the Department of Pediatrics of the Hirosaki University Hospital. After pediatric surgeons performed a total resection of his right kidney and a histopathologic examination, he was diagnosed as having clear-cell renal cell carcinoma. After surgery, he was treated with chemotherapy, and his general condition gradually improved. However, he then exhibited general malaise and was diagnosed as having secondary AML in May 2012. Although chemotherapy treatment for the AML was once again successful, subsequent tumor lysis syndrome, DIC, intracerebral hemorrhage, and secondary renal and heart failures led to severe deterioration of his general condition.

An ultrasound examination performed by his pediatricians detected bilateral intraocular protrusions, and he was referred to our ophthalmology clinic. We were unable to measure his visual acuity at the first examination, as he had been sedated for general treatment. Subsequent fundus examinations did show intraocular protrusions, and he was diagnosed with bilateral subretinal or choroidal hemorrhages. A few weeks later, bilateral dense vitreous hemorrhages occurred, his fundi became totally obscured, and he became blind. Electroretinograms (ERG) were extinguished bilaterally, and A/B-mode ultrasonographies revealed bilateral combined vitreous hemorrhage and hemorrhagic retinal detachment (fig. 1).

After intensive pediatric treatment, his general condition improved to the point where he was well enough to receive transplantation of umbilical cord blood stem cells in September 2012. Transplanted cord blood stem cells were successfully engrafted, and his general condition continued to improve. Because both his parents and the pediatricians wanted to treat his blindness with surgery, we agreed to perform vitrectomy associated with lens extraction and intraocular lens (IOL) implantation in his left eye. The left eye was chosen for the surgery as it still demonstrated a sluggish direct light reflex, while his right eye had completely lost the direct light reflex.

Although his visual acuity showed no light perception bilaterally, preoperative ocular examinations revealed that a sluggish direct light reflex remained in his left eye and that bilateral dense massive vitreous hemorrhages could still be observed (fig. 2). However, ultrasonographic examinations showed that the previously detected hemorrhagic retinal detachments (fig. 1) were no longer observable.

In December 2012, the patient underwent 25-gauge pars plana vitrectomy combined with phacoemulsification and aspiration (PEA). We first prepared surgical ports for the 25- 
Kudo et al.: A Case of Childhood Vitrectomy Performed for Dense Vitreous Hemorrhage Secondary to Leukemia Therapy and Tumor Lysis Syndrome

gauge vitrectomy and then uneventfully performed PEA before starting the vitrectomy procedure. After removal of the dense vitreous hemorrhage, we observed that the peripheral retinal color was mottled, similar to that which is seen after the complete absorption of a subretinal hemorrhage. Surprisingly, with the exception of numerous fine retinal folds, the retinal color in the posterior pole appeared almost normal, and the optic disc was not pale. Because we detected a putative perforating retinal hole in the mid-peripheral portion, laser photocoagulation was applied around the lesion. Next, an IOL was implanted in the bag, and a posterior capsulotomy was performed. We then closed all surgical incisions. No surgical complications occurred during or after the operation. Although the postoperative visual acuity remained at light perception for 3 months, it gradually started to improve 4 months postoperatively. The condition of the fundus appeared to be almost the same as that seen at the end of surgery (fig. 3a). An optical coherence tomography examination of the left eye indicated that while the shape of the central fovea was normal, the external limiting membrane was absent, and the inner segment ellipsoid line was extremely thin and not continuous under the fovea (fig. 3b). In September 2013, the corrected visual acuity in his left eye improved to 0.1 .

\section{Discussion}

We evaluated and then performed a vitrectomy in a child with severe massive vitreous hemorrhage due to leukemia or secondary to chemotherapy for leukemia. Retinal hemorrhages, pre- and subretinal hemorrhages, cotton wool spots, optic disc edema, and invasion of neoplastic cells, among others, are some of the various ocular complications that have been reported for leukemia over the years [1]. However, bilateral massive vitreous hemorrhages are extremely rare [2-6]. Tanji et al. [7] have reported adult cases with vitreous hemorrhages that were related to leukemia. In such cases, a decrease in erythrocytes and/or platelets can lead to an increase in capillary permeability, subsequently allowing the erythrocytes to easily leak out to extravascular spaces. In addition, the decrease in leukocytes causes a reduction in the immune capacity and an increase in the vulnerability to infections, which may ultimately cause vascular inflammation and lead to an increased capillary permeability. On the other hand, DIC induces coagulopathy and impairment of the repair mechanisms of vessels, which can then exacerbate the hemorrhagic complications [8]. Furthermore, retinal ischemia due to leukemic retinopathy or invasion of tumor cells may cause vitreous hemorrhage. In our case, the patient developed acute renal failure, which may also have induced dysfunction of the platelets. Although his intracerebral hemorrhage showed a massive cerebral stromal hematoma associated with its rupture to the lateral ventricle but without subarachnoid hemorrhage, there might still be a possibility that the intraocular hemorrhage occurred by a mechanism similar to Terson syndrome.

As mentioned above, there are various causes of vitreous hemorrhage known to be associated with leukemia. In the present case, bilateral massive vitreous hemorrhage was considered to be the most probable mechanism due to a reduction of platelets caused by chemotherapy and DIC. In this case, we were able to perform a combination of PEA, pars plana vitrectomy, and implantation of IOL without any severe complications. The red blood cells massively occupied the vitreous space adjacent to the posterior lens capsule (fig. 2), so that we decided to remove the crystalline lens to maintain fundus visibility during surgery. Because the patient had reached the age of 4 years, his axial length was approximately 22.0 $\mathrm{mm}$, which provided sufficient space for us to safely perform these surgical procedures. Fortunately, complete posterior vitreous detachment had also occurred, and there was neither 
Kudo et al.: A Case of Childhood Vitrectomy Performed for Dense Vitreous Hemorrhage Secondary to Leukemia Therapy and Tumor Lysis Syndrome

retinal detachment nor retinal proliferation and adhesion. Moreover, there was no continuous hemorrhage in this case. As a result, it was not necessary for us to make a retinal break or to inject silicone oil into the vitreous cavity. If we had encountered any of the abovementioned complications, the surgery would have been more difficult to perform.

Although the preoperative visual acuity was classified as no light perception and associated with extinguished ERG responses, and only a sluggish light reflex was noted, the patient's visual acuity eventually improved to 0.1 in his left eye. The results show that the use of vitrectomy can be considered for a child with severe vitreous hemorrhage, provided that the general condition of the child is good enough to undergo surgery, and 'no perception of light' does not necessarily mean complete loss of vision in children.

\section{References}

1 Duke-Elder S: The leucaemias. Blood diseases; in Duke-Elder S (ed): System of Ophthalmology X. London, Henry Kimpton, 1967, pp 387-393.

-2 Krebs W, Metz O, Plenert W: Ocular symptoms and leukemia in childhood. Klin Monbl Augenheilkd 1982;181:407-408.

-3 Ohkoshi K: Ocular manifestations of childhood leukemia (in Japanese). Nihon Ganka Gakkai Zasshi 1988;92:1787-1796.

4 Marshall RA: A review of lesions in the optic fundus in various diseases of the blood. Blood 1959;14:882891.

5 Reddy SC, Menon BS: A prospective study of ocular manifestations in childhood acute leukemia. Acta Ophthalmol Scand 1998;76:700-703.

6 Fujita A, Tsuchida Y, Shiraga F, Ohtsuki H, Sakaguchi N, Takei Y: Vitreous hemorrhage in a case of acute myeloblastic leukemia (in Japanese). Rinsho Ganka 2001;55:909-912.

7 Tanji K, Nakano K, Funatsu H, Kosaka T, Choushi K, Arihiro K, Iwato K: A case of vitreous hemorrhage with acute myeloblastic leukemia. Folia Ophthalmol Jpn 1994;45:458-462.

-8 Lewis K, Herbert EN, Williamson TH: Severe ocular involvement in disseminated intravascular coagulation complicating meningococcaemia. Graefes Arch Clin Exp Ophthalmol 2005;243:1069-1070.

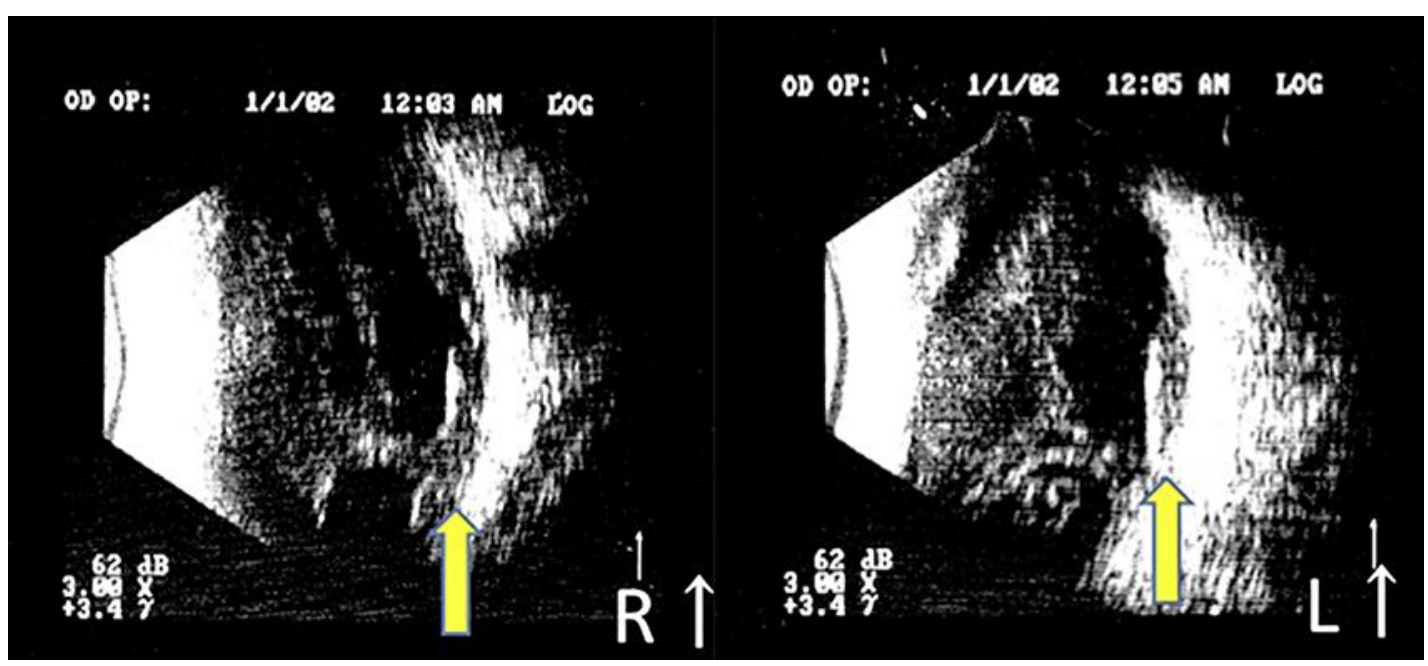

Fig. 1. B-mode ultrasound examinations revealed vitreous hemorrhages and subretinal hyperechoic lesions suggesting bilateral hemorrhagic retinal detachments (large arrows). $\mathrm{R}=$ Right eye; $\mathrm{L}=$ left eye. The small arrows point towards the upper side. 


\section{Case Reports in \\ Ophthalmology}

\begin{tabular}{l|l}
\hline \multicolumn{2}{l|}{ Case Rep Ophthalmol 2015;6:34-38 } \\
\hline DOI: $10.1159 / 000374088$ & $\begin{array}{l}\text { C } 2015 \text { S. Karger AG, Basel } \\
\text { www.karger.com/cop }\end{array}$ \\
\hline
\end{tabular}

Kudo et al.: A Case of Childhood Vitrectomy Performed for Dense Vitreous Hemorrhage Secondary to Leukemia Therapy and Tumor Lysis Syndrome

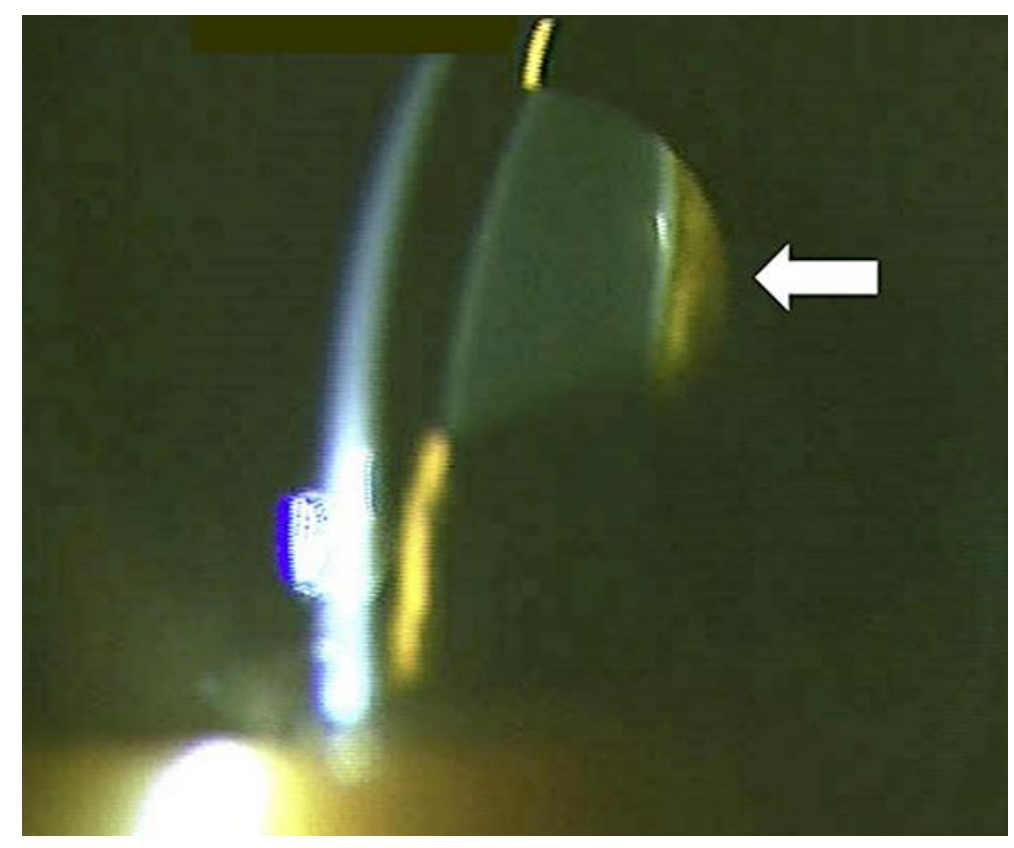

Fig. 2. Slit-lamp findings for the left eye. Dense vitreous hemorrhage was detected (arrow).
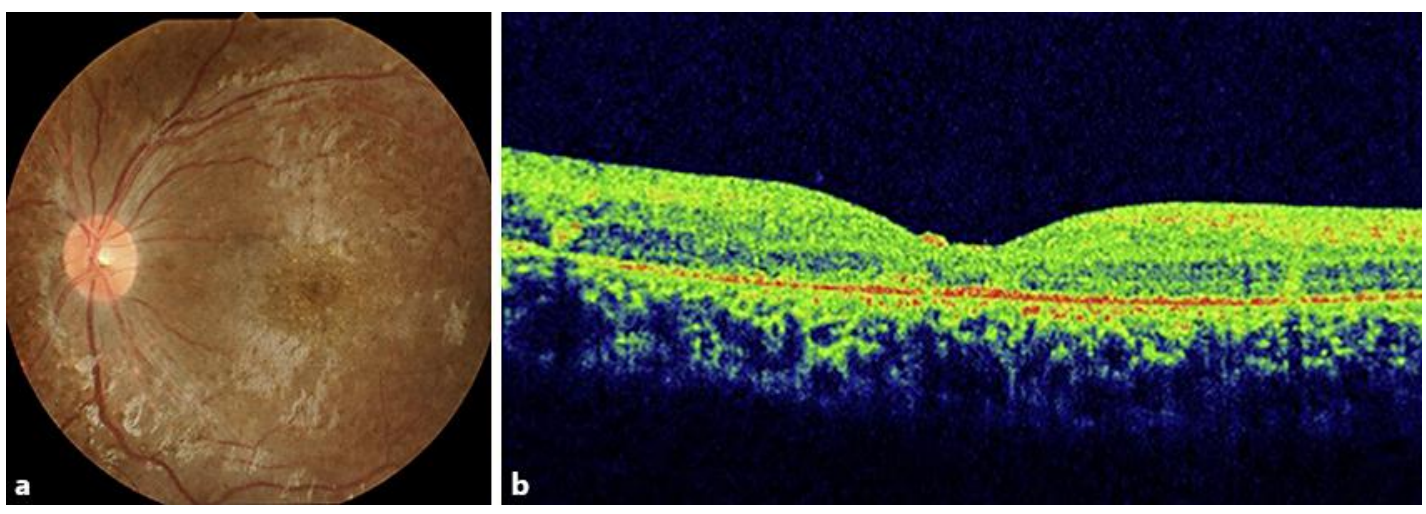

Fig. 3. Postoperative findings. a Fundus photography for the left eye 2 months after surgery. The optic disc and the macula exhibited a normal color. Numerous fine retinal folds were also observed. b Optical coherence tomography findings for the left eye. While the shape of the central fovea was normal, the external limiting membrane was absent, and the inner segment ellipsoid line was extremely thin and not continuous under the fovea. 